\title{
The Intraosseus Tumor - A Potential Pitfall for the Arthroplasty Surgeon: A Case Report.
}

Julian Fromm ( $\boldsymbol{\sim}$ Julian.Fromm@med.uni-muenchen.de )

LMU: Ludwig-Maximilians-Universitat Munchen https://orcid.org/0000-0003-2973-764X

Christof Birkenmaier

LMU: Ludwig-Maximilians-Universitat Munchen

Sophia Goller

LMU: Ludwig-Maximilians-Universitat Munchen

Alexander Klein

LMU: Ludwig-Maximilians-Universitat Munchen

Thomas Knoesel

LMU: Ludwig-Maximilians-Universitat Munchen

Volkmar Jansson

LMU: Ludwig-Maximilians-Universitat Munchen

Hans Roland Dürr

LMU: Ludwig-Maximilians-Universitat Munchen

\section{Research Article}

Keywords: Intraosseus Tumor, Cyst, Myxoid, Chondrosarcoma, Arthroplasty, Case Report

Posted Date: October 25th, 2021

DOI: https://doi.org/10.21203/rs.3.rs-966709/v1

License: (c) (1) This work is licensed under a Creative Commons Attribution 4.0 International License.

Read Full License 
The intraosseus tumor - a potential pitfall for the arthroplasty surgeon: A case report.

Fromm, Julian ${ }^{1}$ (Julian.Fromm@med.uni-muenchen.de) - corresponding author, Birkenmaier, Christof ${ }^{1}$ (Christof.Birkenmaier@med.uni-muenchen.de),

Goller, Sophia² (Sophia.Goller@med.uni-muenchen.de),

Klein, Alexander ${ }^{1}$ (Alexander.Klein@med.uni-muenchen.de),

Knösel, Thomas ${ }^{3}$ (Thomas.Knoesel@med.uni-muenchen.de),

Volkmar Jansson ${ }^{1}$ (Volkmar.Jansson@med.uni-muenchen.de)

Dürr, Hans Roland ${ }^{1}$ (Hans_Roland.Duerr@med.uni-muenchen.de)

${ }^{1}$ Musculoskeletal Oncology, Department of Orthopedics, Physical Medicine and Rehabilitation, University Hospital, Ludwig-Maximilians-University, Campus Grosshadern, Marchioninistr. 15, D-81377 Munich, Germany

${ }^{2}$ Department of Radiology, University Hospital, Ludwig-Maximilians-University, Campus Grosshadern, Marchioninistr. 15, D-81377 Munich, Germany

${ }^{3}$ Institute of Pathology, Faculty of Medicine, University Hospital, Ludwig-MaximiliansUniversity, Campus Grosshadern, Marchioninistr. 15, D-81377 Munich, Germany 


\section{Abstract}

Background: Although rare, the annual incidence of intraosseus sarcomas is up to 2 per 100.000 population with a peak in the sixth decade of life. Due to their slow growth and because they are often asymptomatic for a long period of time, they can be misinterpreted as a benign bony lesion. Particularly, if the tumor shows a high myxoid signal on MRI, the lesion might be mistaken for a bone cyst.

Case presentation: We present the case of a 70-year-old female complaining of pain in her left knee. Initial imaging showed a grade 3 osteoarthritis, according to the Kellgren-Lawrence classification system, and a cystic lesion of the distal femur. The patient received a total knee endoprothesis and five years after the index surgery, she presented again with pain in her left knee after a fall. Radiographs showed an expansile lesion of the distal femur. A biopsy was performed and the lesion proved to be a chondrosarcoma with myxoid pattern. After en bloc-resection, a megaendoprosthesis was implanted in a palliative approach. Four years later, the patient died without local recurrence due to metastatic disease from an adenocarcinoma of unknown origin.

Conclusion: Malignant tumors should be taken into consideration whenever imaging shows an expansile lesion of the bone, even when they are painless, well delimited and slowly or not at all growing. We recommend a biopsy to be performed before performing an arthroplasty in such cases.

Keywords

Intraosseus Tumor; Cyst; Myxoid; Chondrosarcoma; Arthroplasty; Case Report 
Accounting for approximately $20 \%$ of all bone sarcomas, chondrosarcoma (CS) is the third most frequent primary skeletal malignancy

[1]. Chondrosarcomas constitute a heterogeneous group of tumors, all characterized by the production of cartilaginous matrix.

The histological subtype mainly influences the outcome of CS. Central (conventional) chondrosarcoma represents about $85 \%$ of this group [2], followed by dedifferentiated chondrosarcoma, which represents about $10-15 \%$ of all CS, and other rare subtypes like mesenchymal and clear cell CS [2, 3].

Conventional chondrosarcoma is typically characterized by its slow growth. Common symptoms are gradually increasing pain or swelling $[4,5]$. In most cases, this entity is diagnosed in the fifth to seventh decade of life, with a slight preference towards males. The preferential sites for CS are the hip and pelvis, followed by the shoulder and the knee [5]. The extraskeletal myxoid chondrosarcoma $(E M C)$ is a rare tumor of uncertain differentiation characterized by abundant myxoid matrix, which historically carries the name chondrosarcoma but, in contrast to the other chondrosarcomas, has no origin in cartilaginous tissue. However, extensive myxoid changes can be seen in conventional central $\mathrm{CS}$ as well and therefore, the differential diagnosis between these entities can be challenging [2].

We present the case of a conventional central CS with a myxoid growth pattern, arising from the left distal femur. At another hospital, this tumor was misinterpreted as a simple bone cyst and therefore neglected during the preoperative planning and implantation of a knee endoprothesis, resulting in an extensive extracompartimental spread of the tumor. The aim of this case report is to raise the awareness for CS and the difficulty and pitfalls associated with diagnosing this lesion. 
A 72-year-old female presented to another institution with pain in her left knee. Advanced osteoarthritis was evident on radiographs and a cement-augmented total knee arthroplasty (TKA) was performed. Before arthroplasty, an osteolytic lesion in the left distal femur was documented and falsley interpreted to be a simple bone cyst, based on an incorrect interpretation of MRI (Fig. 1). A "fluid-like" effusion from the lesion into the surgical site was described in the operative report of the TKA. During follow-up, the patient was pain-free for four years. However, postoperative radiographs (Fig. 2) showed a gradually developing local recurrence of the bone lesion, which at the time was falsely interpreted as aseptic loosening of the femoral component.

Five years after the index surgery, the patient fell and presented to another hospital because of pain in her left knee. Again, radiographs were done and a fracture was excluded, but an osseus distension of the middle and distal femur with cortical thinning and structural alteration was apparent (Fig. 3). Consequently the female was referred to our institution. On specific questioning, it turned out that the patient had felt pain and some tension in her left leg for the last year, which she had considered a "muscle ache“ and therefore no diagnostic measures had been initiated. At the time of presentation to our institution, the patient was mobile on crutches because of the pain.

In the further work-up thoracic and abdominal CT scan was performed and no primary tumor or signs of metastatic disease were seen. Additionally, contrast-enhanced MRI of the left leg was done, which showed an extra- and intramedullary lesion extending from the femoral component of the TKA to the proximal third of the left femur (Fig. 4). A tru-cut biopsy of the lesion was obtained and histopathological analysis revealed a low-grade conventional CS. However, due to the aggressive growth pattern of the lesion, a wide resection of the lesion was planned. Due to tumor contamination of bone and adjacent soft tissues, performing a wide resection would have required an amputation of the leg. Considering the patient's age, we recommended a resection of the distal femur, the explantation of the tibial component of the knee endoprothesis and the implantation of a cemented megaendoprothesis in a palliative approach. The intraoperative specimen confirmed the initial diagnosis of a conventional G2-chondrosarcoma with high cellularity, extensive myxoid matrix and moderate atypical nuclei (Fig. 5).

The postoperative course was uncomplicated, and the patient was discharged. Six months after surgery, she was pain-free, mobile using a wheeled walker, and had no problems with daily life activities. Follow-up of the CS consisted of contrast-enhanced MRI of the left leg every three months during the first year, every six months during the second year and yearly after. In addition, thoracic CT for two years every six months and yearly afterwards was recommended.

Two years after tumor resection, the patient again presented with pain in her left knee. Radiographs showed loosening of the femoral stem (Fig. 6). Consecutively the stem had to be exchanged, which again was unproblematic. Tumor was not encountered during this procedure. Postoperatively, the patient again underwent rehabilitation therapy and continued walking with a wheeled walker without major disabilities. 
Four years after tumor resection, multiple nodular liver lesions were detected coincidentally in the follow-up examination, which was performed at a peripheral hospital. Liver biopsy was performed and metastatic disease due to an adenocarcinoma of unknown origin was diagnosed. The primary carcinoma could not be identified, however, and the patient was discharged home with palliative care on her own request and passed away one month later. 


\title{
Discussion
}

Both, EMC and conventional central CS often present with pronounced myxoid matrix $[2,5]$. While conventional CS by definition arises in the bone, EMC of the bone is rare, and the current literature manly consists of case reports [6]. However, there are reports of EMC arising in locations commonly entered during arthroplasty surgery, like the proximal or distal femur [6-8].

While these lesions are usually well recognized on CT- or MRI, imaging diagnosis might be difficult on conventional radiographs alone, particularly in small lesions. This can be a problem, especially, since the preoperative planning prior to the implantation of an endoprosthesis often consists solely of conventional radiographs. There are numerous reports on incidental findings of sarcomas, carcinomas and other tumors in the process of arthroplasty [9-13]. Due to its myxoid matrix, CS as well as EMC might be mistaken for a juxta-articular myxoma (JAM), a generally benign and common lesion, which typically occurs in and around big joints, especially the knee joint (88\%)[14-16], or a common bone cyst. Since CS and EMC are slow growing lesions, which are often relatively painless for a long period of time and usually appear around the fifth to seventh decade of life, these tumors should be in the back of the mind in arthroplasty surgery. In our case, the lesion was noticed preoperatively and therefore MRI was performed, however, the lesion was falsely considered to be a benign cyst, a diagnostic mistake, which might have been avoided if the MRI would have been contrast-enhanced. Intraoperatively, a histopathological analysis of a sample of the lesions could have helped diagnosing the CS at that time.

\section{Conclusion}

In conclusion, we share this case to raise awareness for intraosseous tumors, i.e., EMC and conventional central CS. We recommend not to forgo the appropriate diagnostic work-up in situations with intraosseus cystic or other lesions prior to a planned arthroplasty, even when the lesion appears painless and are slowly or not at all growing at all.

\author{
Abbreviations \\ CS - chondrosarcoma \\ EMC - extraskeletal myxoid chondrosarcoma \\ TKA - total knee arthroplasty \\ $\mathrm{MRI}$ - magnetic resonance imaging \\ CT - computed tomography \\ JAM - juxta-articular myxoma
}


Figures:

Figure 1:

(a-b) Coronal and sagittal MRI of the left knee (proton-density weighted fat-saturated images) showing an epimetaphyseal cystic lesion in the distal femur with anterior cortical destruction $\left({ }^{*}\right)$.

Figure 2:

(a-b) Postoperative radiographs 3 years after index surgery showing the lesion growing in the diaphyseal femur. At that point of time, it was misinterpreted as initial aseptic component loosening.

Figure 3:

(a-c) Radiographs taken at the day of admission at our hospital 5 years after the index surgery. Osseus expansion of the femoral metaphysis proximal to the femoral component with extensive cortical thinning and irregular appearance of trabecular structure is obvious.

Figure 4:

(a-b) Axial and coronal MR images of the left leg (T1 weighted contrast-enhanced sequences), demonstrating the intra- and extracompartimental growth of the lesion with increased contrast uptake at its margins.

Figure 5:

(a) Chondrosarcoma (G2) with atypical chondrocytes (10x magnification, HE staining).

(b) Chondrocytes with invasive growth pattern between bone trabeculae (20x magnification, HE staining)

Figure 6:

(a-b) Radiographs revealed signs of loosening of the femoral component two years after megaprosthesis implantation. 
Ethics approval and consent to participate

The analysis of the patient data and this case report were approved by the institutional ethics committee of our faculty (\#17-891)

Consent for publication

Written informed consent was obtained from the patient for publication of this case report and any accompanying images. A copy of the written consent is available for review by the Editor-in-Chief of this journal.

Availability of data and materials

Not applicable

Competing interests

The authors declare they have no competing interests.

Funding

There was no funding concerning this case report.

Acknowledgements

Not applicable

Author's contributions

JF wrote the case report, HRD treated the patient and provided the data, CB reviewed and corrected the manuscript, AK proof-read the manuscript, TK provided the histological microphotographs and the histolopathological background, SG provided the radiological imaging and helped with the radiological analysis. 
1. Doyle LA: Sarcoma classification: an update based on the 2013 World Health Organization Classification of Tumors of Soft Tissue and Bone. Cancer 2014, 120(12):1763-1774.

2. Hogendoom EA: Chondrosarcoma (grades I-III) including primary and secondary variants and periosteal chondrosarcoma. In: WHO Classification of Tumours of Soft Tissue and Bone. Volume 4, edn. Edited by Fletcher CDM BJ, Hogendoorn PCW, Mertens F,. Lyon; 2013: 264-269.

3. Gelderblom H, Hogendoorn PC, Dijkstra SD, van Rijswijk CS, Krol AD, Taminiau $\mathrm{AH}$, Bovee JV: The clinical approach towards chondrosarcoma. Oncologist 2008, 13(3):320-329.

4. Karpik M, Reszec J: Low Grade Chondrosarcoma - Epidemiology, Diagnosis, Treatment. Ortop Traumatol Rehabil 2018, 20(1):65-70.

5. D.R. Lucas GS: World Health Organization Classification of Tumours. Pathology and Genetics of Tumours of Soft Tissue and Bone. Lyon: IARC Press; 2013.

6. Finos L, Righi A, Frisoni T, Gambarotti M, Ghinelli C, Benini S, Vanel D, Picci P: Primary extraskeletal myxoid chondrosarcoma of bone: Report of three cases and review of the literature. Pathol Res Pract 2017, 213(5):461-466.

7. Enzinger FM, Shiraki M: Extraskeletal myxoid chondrosarcoma. An analysis of 34 cases. Hum Pathol 1972, 3(3):421-435.

8. Shao R, Lao IW, Wang L, Yu L, Wang J, Fan Q: Clinicopathologic and radiologic features of extraskeletal myxoid chondrosarcoma: a retrospective study of 40 Chinese cases with literature review. Ann Diagn Pathol 2016, 23:14-20.

9. Kahn DG, Blazina ME: Incidental metastatic mammary carcinoma in a total knee arthroplasty patient. Clin Orthop Relat Res 1993(295):142-145.

10. Savvidou OD, Chloros GD, Koutsouradis P, Megaloikonomos PD, Skarpidi E, Papagelopoulos PJ: Synovial Sarcoma Complicating Total Knee Arthroplasty. Clin Orthop Surg 2017, 9(4):547-552.

11. Durr HR, Stabler A, Maier M, Refior HJ: Pigmented villonodular synovitis. Review of 20 cases. J Rheumatol 2001, 28(7):1620-1630.

12. Lei P, Sun R, Liu H, Zhu J, Wen T, Hu Y: Prognosis of Advanced Tenosynovial Giant Cell Tumor of the Knee Diagnosed During Total Knee Arthroplasty. J Arthroplasty 2017, 32(6):1850-1855.

13. Visser J, Busch VJ, de Kievit-van der Heijden IM, ten Ham AM: Non-Hodgkin's lymphoma of the synovium discovered in total knee arthroplasty: a case report. BMC Res Notes 2012, 5:449.

14. Beggan $C$, Davies $K$, Leader M: Juxta-articular myxoma: an unusual benign mesenchymal lesion, readily mistaken for malignancy. Ir Med J 2014, 107(7):212-213.

15. Meis JM, Enzinger FM: Juxta-articular myxoma: a clinical and pathologic study of 65 cases. Hum Pathol 1992, 23(6):639-646.

16. Somford MP, de Vries JS, Dingemans W, de Jonge M, Maas M, Schaap GR, Bramer JA: Juxta-articular myxoma of the knee. J Knee Surg 2011, 24(4):299-301. 


\section{Figures}

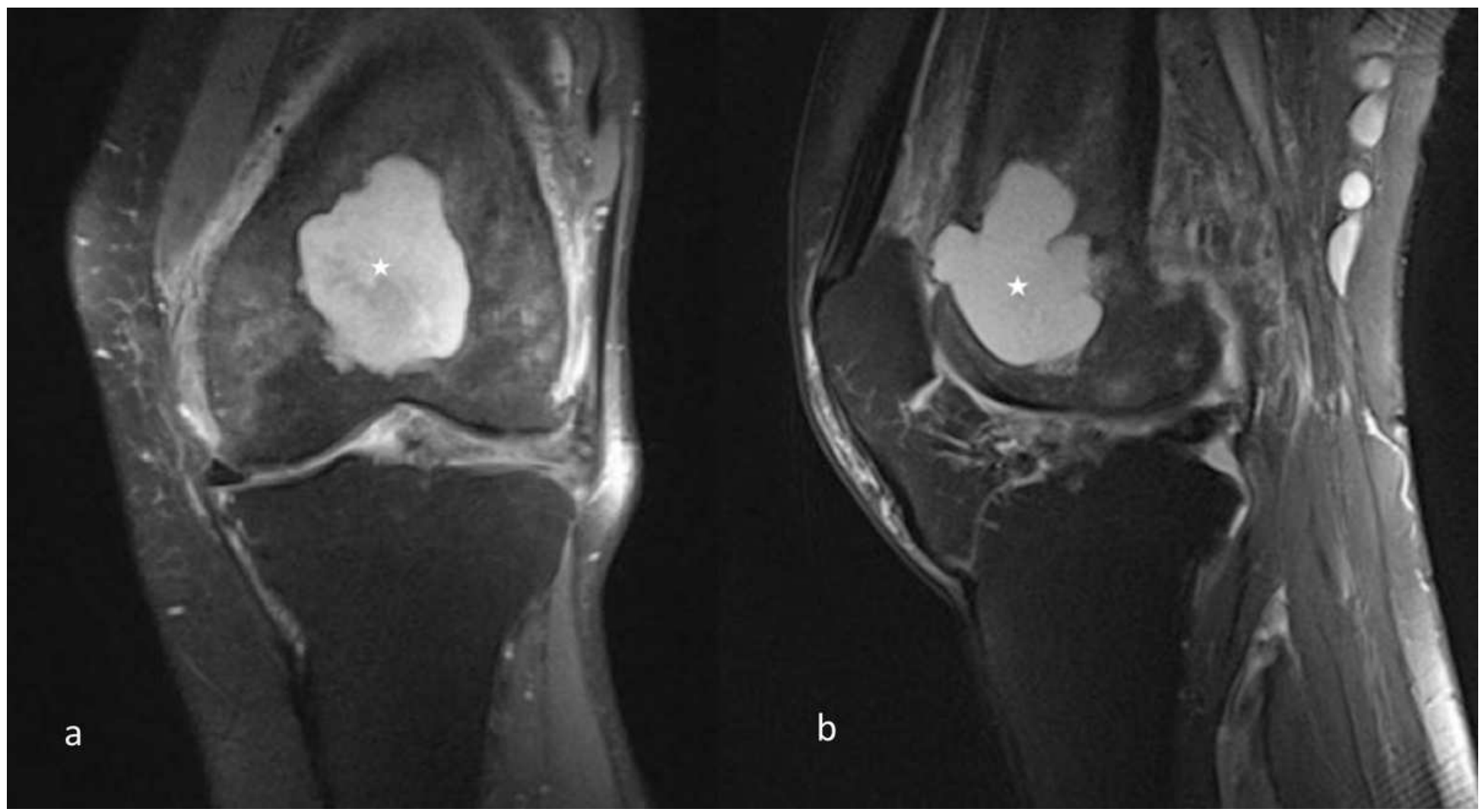

\section{Figure 1}

(a-b) Coronal and sagittal MRI of the left knee (proton-density weighted fat-saturated images) showing an epimetaphyseal cystic lesion in the distal femur with anterior cortical destruction $\left(^{*}\right)$. 

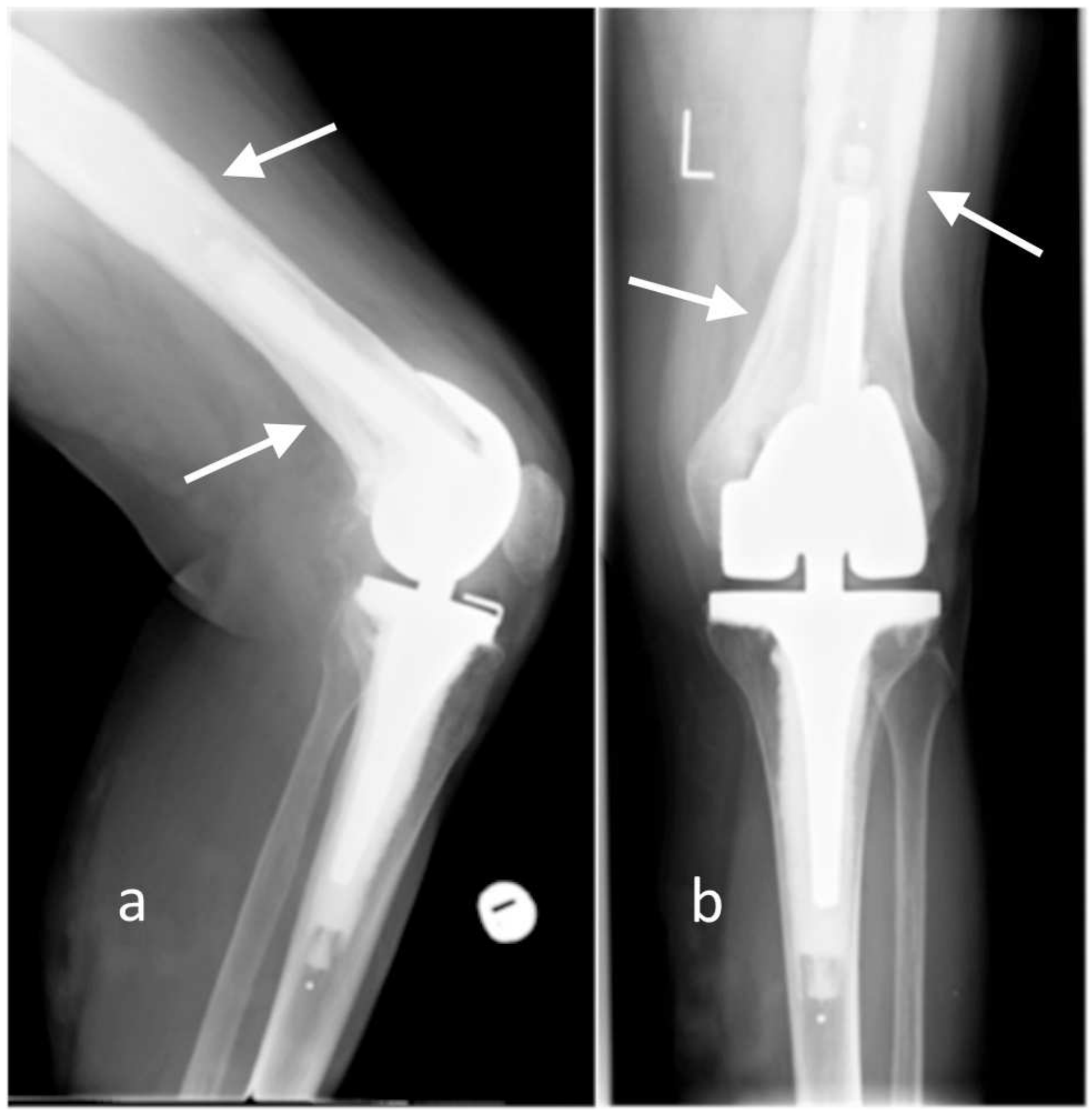

Figure 2

(a-b) Postoperative radiographs 3 years after index surgery showing the lesion growing in the diaphyseal femur. At that point of time, it was misinterpreted as initial aseptic component loosening. 


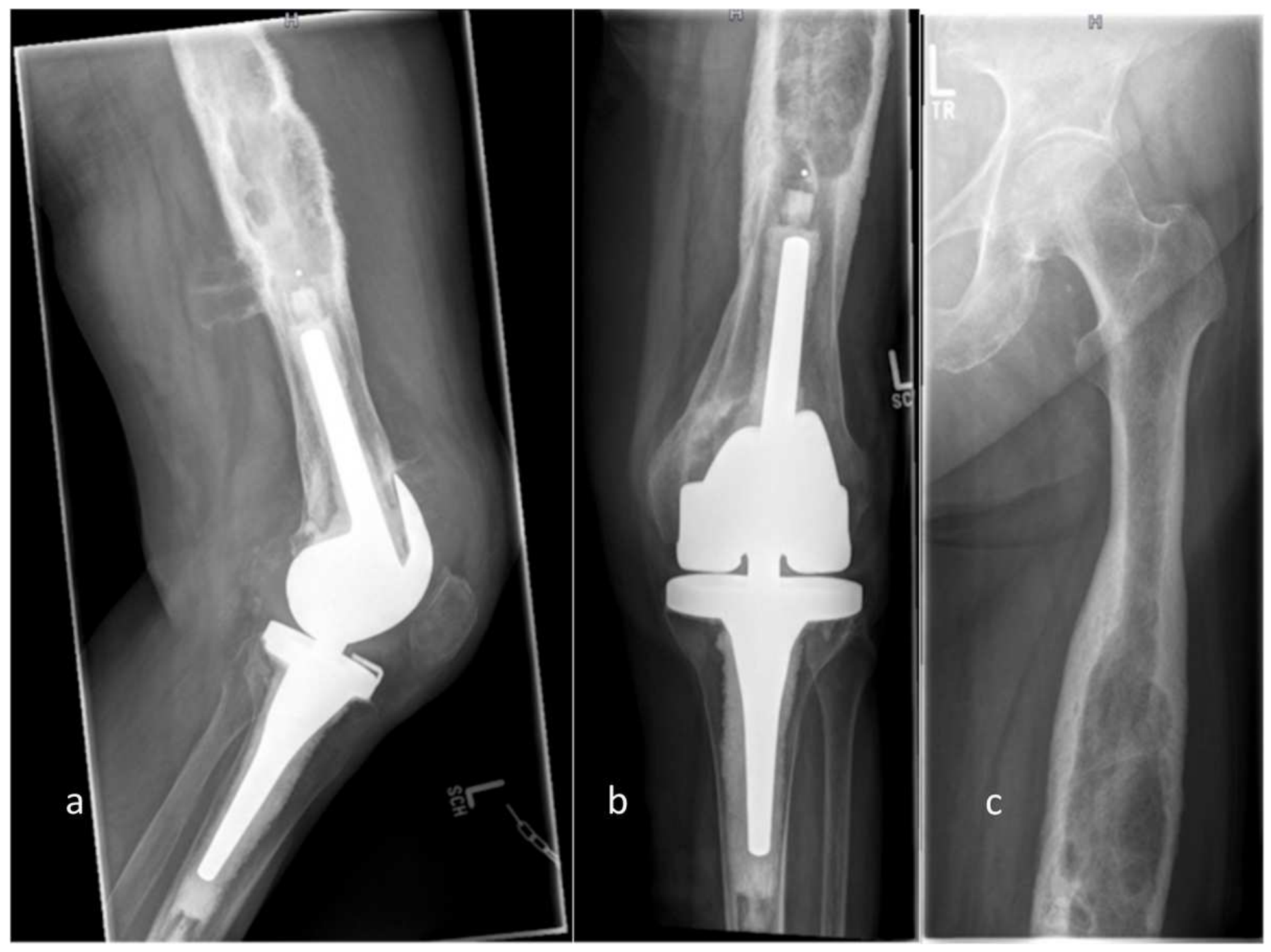

\section{Figure 3}

(a-c) Radiographs taken at the day of admission at our hospital 5 years after the index surgery. Osseus expansion of the femoral metaphysis proximal to the femoral component with extensive cortical thinning and irregular appearance of trabecular structure is obvious. 


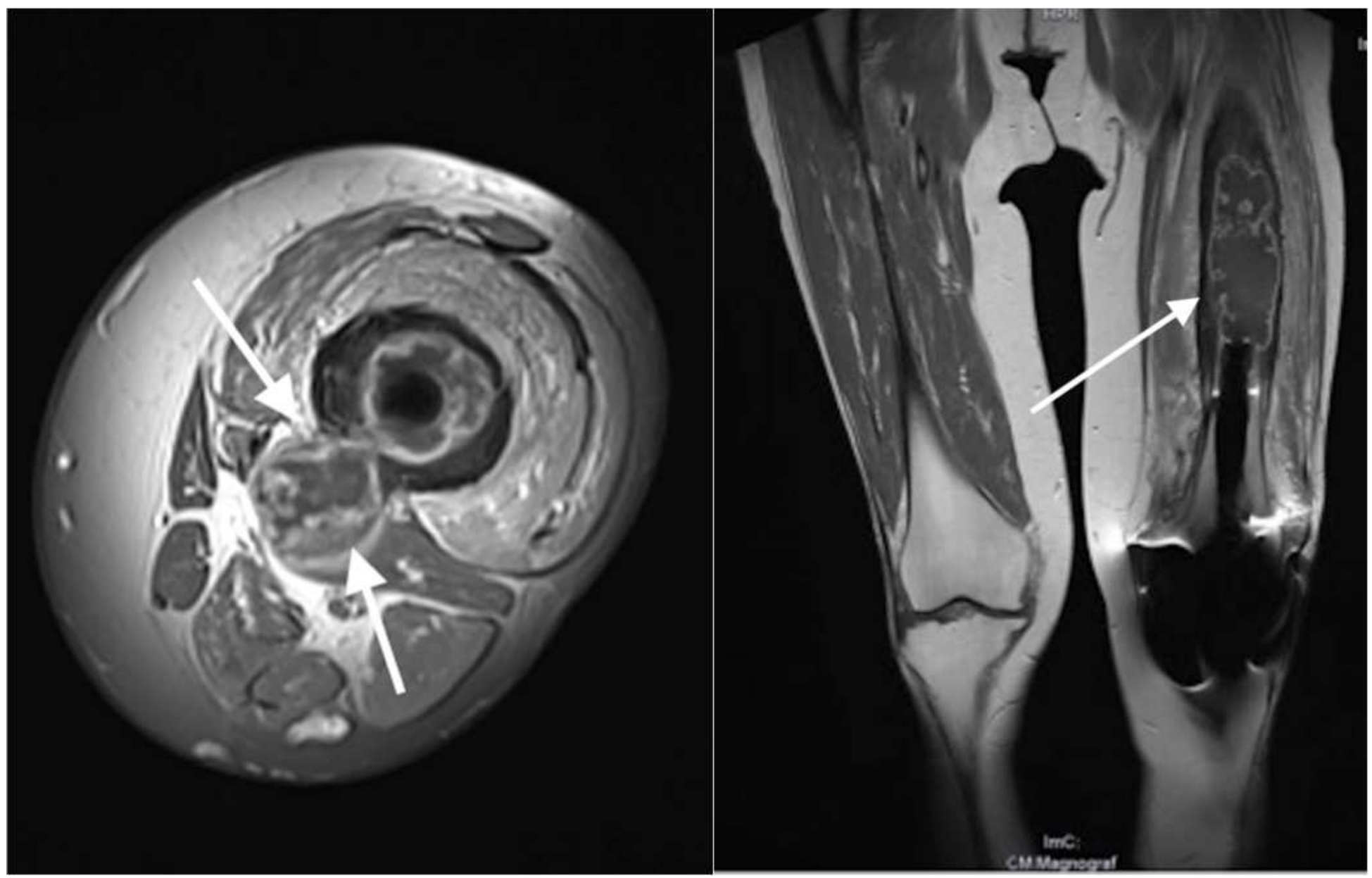

\section{Figure 4}

(a-b) Axial and coronal MR images of the left leg (T1 weighted contrast-enhanced sequences), demonstrating the intra- and extracompartimental growth of the lesion with increased contrast uptake at its margins.

2

\section{Figure 5}

(a) Chondrosarcoma (G2) with atypical chondrocytes (10x magnification, HE staining). (b) Chondrocytes with invasive growth pattern between bone trabeculae (20x magnification, HE staining) 


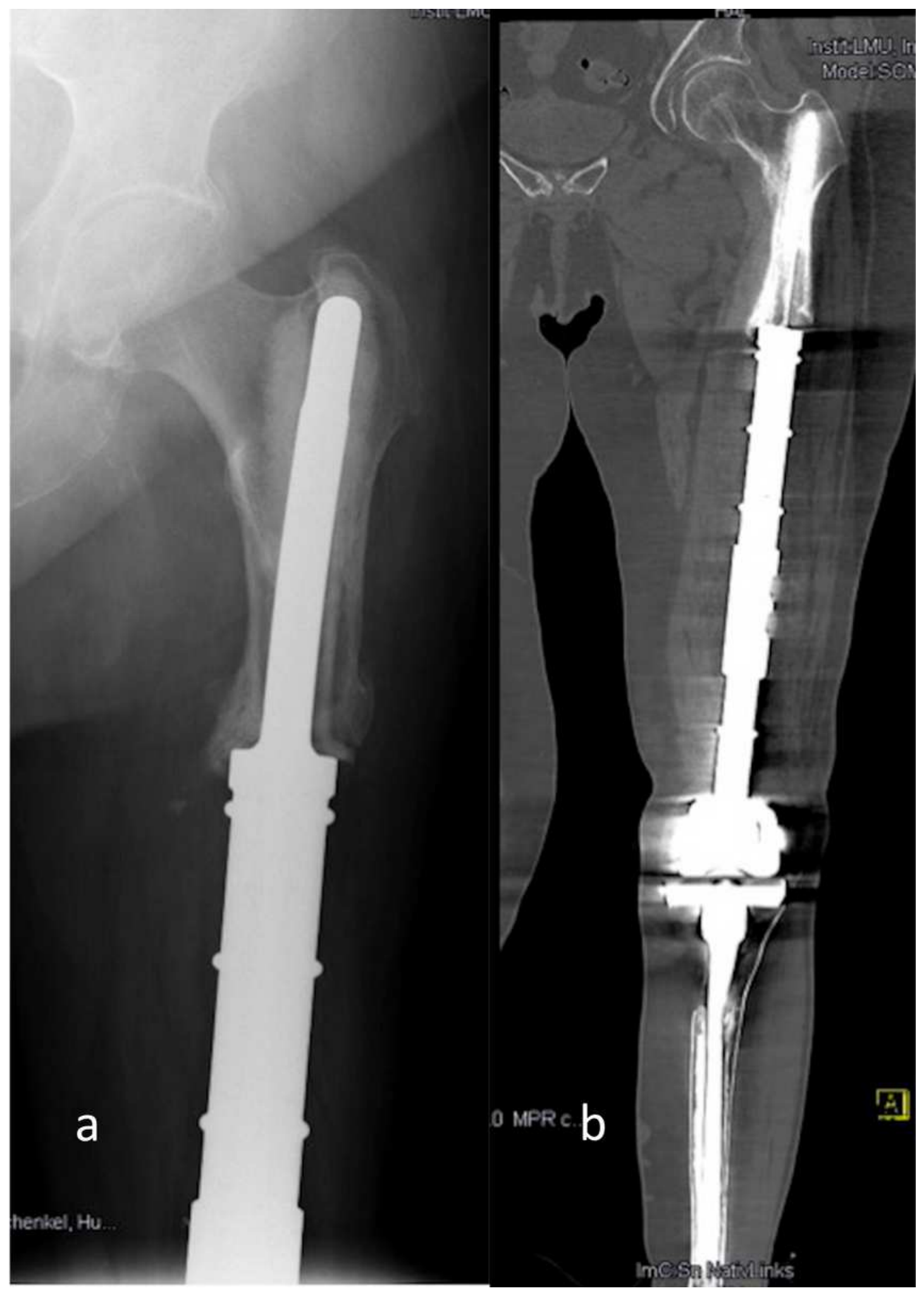

Figure 6

(a-b) Radiographs revealed signs of loosening of the femoral component two years after megaprosthesis implantation.

\section{Supplementary Files}


This is a list of supplementary files associated with this preprint. Click to download.

- CAREchecklistCRFromm.pdf 